\title{
The influence of electrode biasing on plasma confinement in J-TEXT tokamak
}

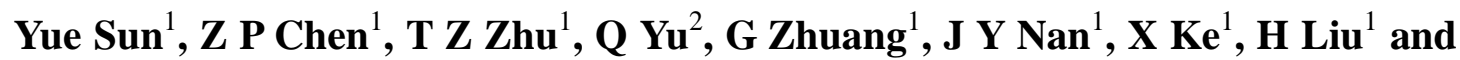 \\ the J-TEXT Team ${ }^{1}$
}

${ }^{1}$ State Key Laboratory of Advanced Electromagnetic Engineering and Technology, College of Electrical and Electronic Engineering, Huazhong University of Science and Technology, Wuhan 430074, People's Republic of China

${ }^{2}$ Max-Planck-Institut für Plasmaphysik, EURATOM Association, 85748 Garching, Germany

E-mail: zpchen@mail.hust.edu.cn

\begin{abstract}
The influence of both positive and negative bias on global and plasma-edge parameters has been comparatively studied with a newly designed electrode biasing system in J-TEXT tokamak. Comparing to the $0 \mathrm{~V}$ bias case, the global particle confinement of plasma is enhanced under bias with both polarities, with the increments of central line-averaged density and soft-X ray emission as well as the reduction of edge $\mathrm{H}_{\alpha}$ radiation level. The suppression of plasma-edge fluctuations and turbulent particle transport are obviously observed under bias, in different degrees with different polarities. The potential fluctuation amplitude is observed to be increased at the vicinity of limiter under positive bias, with the existence of a peaked low frequency mode (LFM) characterized as high coherence and near-zero cross-phase poloidally in the edge region, which is not found in the negative bias case. The poloidal correlation length of turbulence is greatly enhanced under bias with both polarities, and shows a positive correlation with the amplitude of poloidal phase velocity, which is mainly driven by the local $J_{\mathrm{r}} \times B$ torque at plasma edge under bias. The characteristic parameters of intermittent events (i.e. blobs), including amplitude, radial velocity, related particle flux and radial size, are decreased dramatically under bias in the edge region.
\end{abstract}

\section{Introduction}

Although H-mode has been discovered for more than three decades since its first realization on ASDEX tokamak in 1982 [1], understanding the physics underlying, especially the mechanism of L-H transition, is still incomplete and needs more investigations. However, more and more experimental and theoretical results support the robust relationship between the $E \times B$ shear flows and the confinement improvement. 
One of the effective ways to drive the local $E \times B$ shear flows is externally inserting an electrode into the local plasma which is biased with respect to the wall (or the limiter). Since the pioneering experimental work performed on CCT in 1989 [2], the biasing experiments have been carried out on many devices with different magnetic configurations, including tokamaks [3-13], stellarators [14, 15], reversed field pinches [16], and basic plasma devices without closed magnetic field lines $[17,18]$. The most common phenomenon obtained through these experiments was the enhancement of local $E \times B$ shear flows, accompanied with suppression of turbulence and improvement of global confinement [19]. The emergence of $E_{\mathrm{r}}$ bifurcation, which is similar to the L-H transition, was firstly reported on CCT under negative bias [2], and later was also observed in TEXTOR with both positive and negative bias [3]. Once a critical value of electrode current was reached, a sudden transition occurred with an abrupt decrease of radial conductivity as well as a sharp increase of $E_{\mathrm{r}}$ and poloidal velocity, which was followed by a significant improvement especially of the particle confinement. To better understand the physical mechanism of $E_{\mathrm{r}}$ bifurcation with biasing, a theoretical model has been proposed based on charge conservation equation [20]. The results indicate that, the interaction between the two nonlinear terms, i.e. ion bulk viscosity and ion orbit losses, gives rise to the bifurcation phenomenon, and various spatial structures of $E_{\mathrm{r}}$ are possible for the same boundary condition.

Although the confinement improvement is considered as a general character of the biasing experiments, its effectiveness, however, seems to be related to the way of biasing. In ISTTOK, a comparison shows that, the limiter biasing is less efficient in driving the $E \times B$ shear flows and improving confinement than the electrode biasing, and negative biasing has negligible influence on the plasma due to the low electrode current level [8]. However, after introducing a small emissive electrode, the efficiency of negative bias has been promoted greatly [21]. The results obtained in T-10 reveal that, the initial (pre-bias) states of plasma, such as the wall condition, may have an essential influence on the improved confinement performance [9].

Recently, the experimental investigation of the interaction between mean and fluctuating shear flows has become a hot topic in biasing related researches. The level of long-distance correlation (LDC) structures in potential fluctuation, which behaves in the similar way as the zonal flows, was observed to be amplified under positive bias on tokamaks [22-24] and stellarators $[14,15]$. Further studies showed that the LDC strength is sensitive to the polarity of bias [23] as well as magnetic perturbation, and reduces at high-density level [25].

A newly designed electrode biasing system (EBS) has been constructed and performed experimentally in the J-TEXT tokamak recently. The influence of both positive and negative bias on global and plasma-edge parameters has been comparatively studied in this paper. The improvement of global particle confinement and the suppression of plasma-edge turbulent 
particle transport are clearly observed under bias with both polarities. The behavior of potential fluctuation is found to be sensitive to the polarity of bias. The poloidal correlation length of turbulence is increased greatly under bias, and shows a positive correlation with the amplitude of poloidal phase velocity, which is mainly driven by the local $J_{\mathrm{r}} \times B$ torque induced by the biasing. Moreover, some characteristic parameters of blob, including amplitude, radial velocity, related particle flux and radial size are dramatically decreased under bias.

The rest of the paper is arranged as follows: the electrode biasing system and the Langmuir probe diagnostic are introduced in section 2, the main data analysis methods used are described in section 3 , the experimental results including the biasing influence on global confinement and plasma-edge parameters are illustrated in section 4, discussions about common and different characteristics of plasma responses to positive and negative bias are presented in section 5 , and finally a conclusion is given in section 6 .

\section{Experimental setup}

An electrode biasing system (EBS) has been designed and constructed on J-TEXT which is a conventional circular cross-section tokomak [26] with a major radius of $105 \mathrm{~cm}$ and a minor radius of $26.5 \mathrm{~cm}$. To reduce the influence on the plasma, the system is designed to contain a pneumatic driving part, and thus the electrode can reciprocate in a single discharge. The front face of the electrode can reach about $5 \mathrm{~cm}$ inside the limiter $(r \sim 21.5 \mathrm{~cm})$ by an innermost insertion. The bias voltage applied on the electrode with respect to the vacuum chamber wall is provided by a power supply consisting mainly of a capacitor bank. According to the parameters of the power supply, the available range of bias voltage is $-400 \sim+400 \mathrm{~V}$. A current-limiting protection module is added to keep the electrode current drawn under the level of $200 \mathrm{~A}$, which is adjustable according to the practical situations. The signals of electrode current $I_{\text {bias }}$ and voltage $U_{\text {bias }}$ were obtained through the sensors installed near the EBS, and acquired with a frequency of $500 \mathrm{kHz}$. The schematic of EBS is shown in figure 1 (a).

The preliminary biasing experiments were executed in hydrogen discharges with limiter configuration. The discharge parameters were set as follows: the plasma current $I_{\mathrm{p}}=120 \mathrm{kA}$, the toroidal magnetic field $B_{\mathrm{t}}=1.8 \mathrm{~T}$, and the central line-averaged density $\left\langle n_{\mathrm{e}}\right\rangle=$ $(1.5 \sim 2.2) \times 10^{19} \mathrm{~m}^{-3}$. During a discharge, a disk shaped $(1 \mathrm{~cm}$ thick and $4 \mathrm{~cm}$ in diameter) electrode head, which is made of hard graphite, was inserted radially into the plasma through a top port of the tokamak, and reached at the positions $r=22.5 \mathrm{~cm}$ (i.e. about $4 \mathrm{~cm}$ inside limiter). The bias voltage applied during the flat-top phase of plasma current was in the range of $-300 \sim 300 \mathrm{~V}$ for the purpose of avoiding disruption, and the duration of biasing was set as $150 \mathrm{~ms}$. The corresponding volt-ampere characteristic of the biased electrode is shown in 
figure 2. Normally, the electrode current under negative bias is often limited by the ion saturation current, which can be calculated according to the Langmuir probe theory by:

$$
I_{s} \cong 0.49 e n_{e} A_{e f f} C_{s} .
$$

Here, $C_{\mathrm{s}}$ is the sound speed and $A_{\text {eff }}$ is the projection area of the electrode in the direction of the magnetic field. The local plasma parameters at the position of electrode can be estimated approximately as follows: the plasma density $n_{\mathrm{e}} \sim 5 \times 10^{18} \mathrm{~m}^{-3}$, the temperatures $T_{\mathrm{i}} \sim T_{\mathrm{e}} \sim 50 \mathrm{eV}$, and the projection area $A_{\text {eff }}=800 \mathrm{~mm}^{2}$. Thus the theoretical prediction of ion saturation current is about $30 \mathrm{~A}$, which is close to the measured electrode current under $-270 \mathrm{~V}$ bias, as presented in figure 2 .
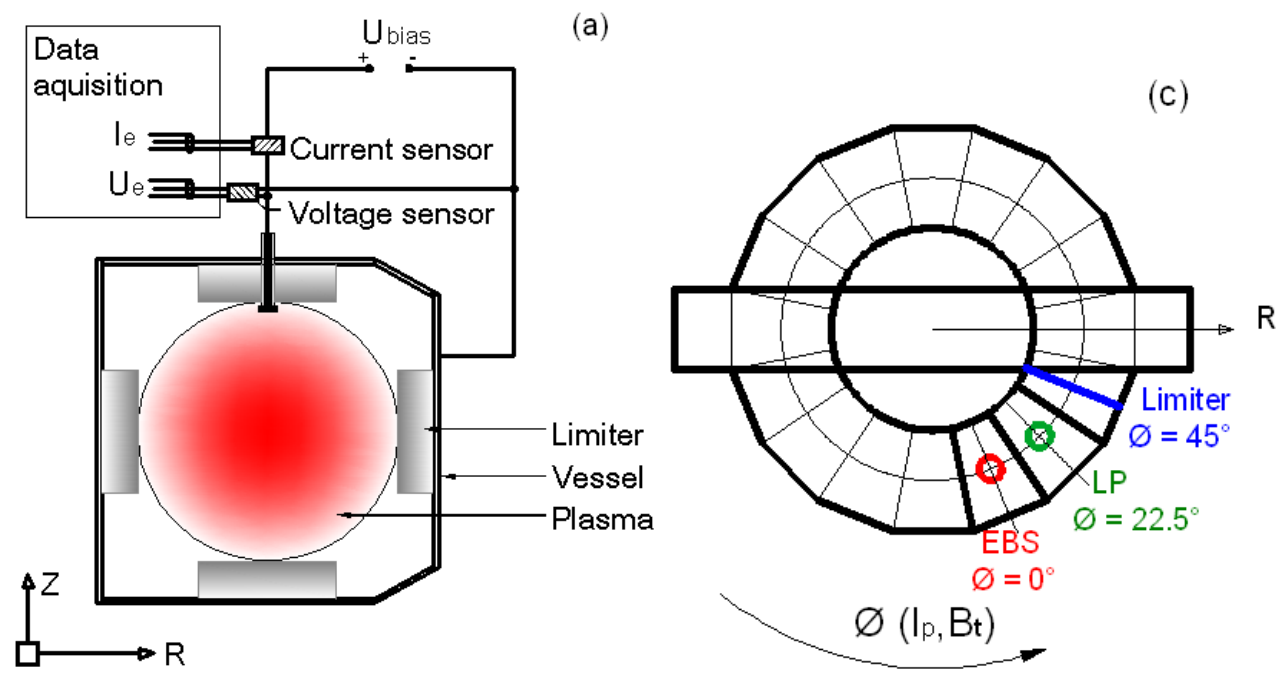

(b)

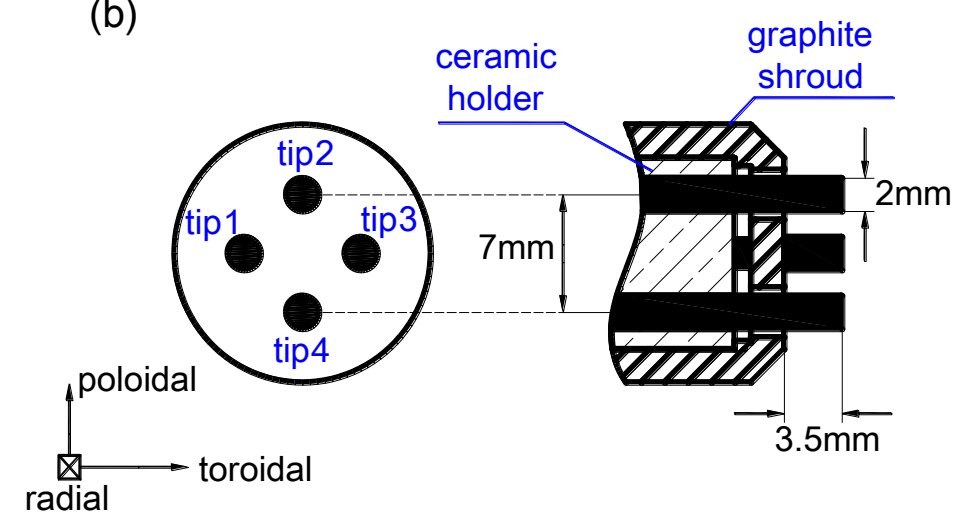

Figure 1. Schematics of (a) electrode bias system (EBS), (b) Langmuir four-tip probe array and (c) the arrangement of the Langmuir probe (LP) system and EBS.

A reciprocating probe system equipped with a Langmuir four-tip probe has been used to monitor the edge plasma behavior in the experiment, which covers a measuring range $r=24.5$ $\sim 29.5 \mathrm{~cm}$. The schematic of the four-tip probe array is presented in figure 1 (b), which is consist of a pair of double probes (tips 1 and 3) and two single probes (tips 2 and 4). The double probes, which are aligned in the toroidal direction, are used to measure the ion 
saturation current $I_{\mathrm{s}}$. While the two single probes, with a poloidal separation of about $7 \mathrm{~mm}$, are used to measure the floating potentials $V_{\mathrm{fl}}$ and $V_{\mathrm{f} 2}$, respectively. The probe data are acquired with a frequency of $2 \mathrm{MHz}$. More information about Langmuir probe (LP) system can be seen in reference [27]. In the late analysis, we find that the double probes (tips 1 and 3) are partially shadowing each other, which is due to the inclined angle between the magnetic field lines and the aligned direction of the probes is too small to avoid shadowing. However, in our opinion, the main influence of shadowing to $I_{\mathrm{s}}$ measurements is the decrease of the absolute values of $I_{\mathrm{s}}$, while the relative value as well as the fluctuating behaviors of $I_{\mathrm{s}}$ should be little influenced. The arrangement of the probe tips remains unchanged under different bias conditions in the experiments, thus the results involving the $I_{\text {sat }}$ measurements in this paper should still be credible.

The directions of plasma current $I_{\mathrm{p}}$ and toroidal field $B_{\mathrm{t}}$ are the same and anticlockwise at top view of the device. As shown in figure 1 (c), both the LP system and EBS are installed on the top ports of J-TEXT, and at toroidal degrees of $22.5^{\circ}$ and $45^{\circ}$ from the limiter, respectively.

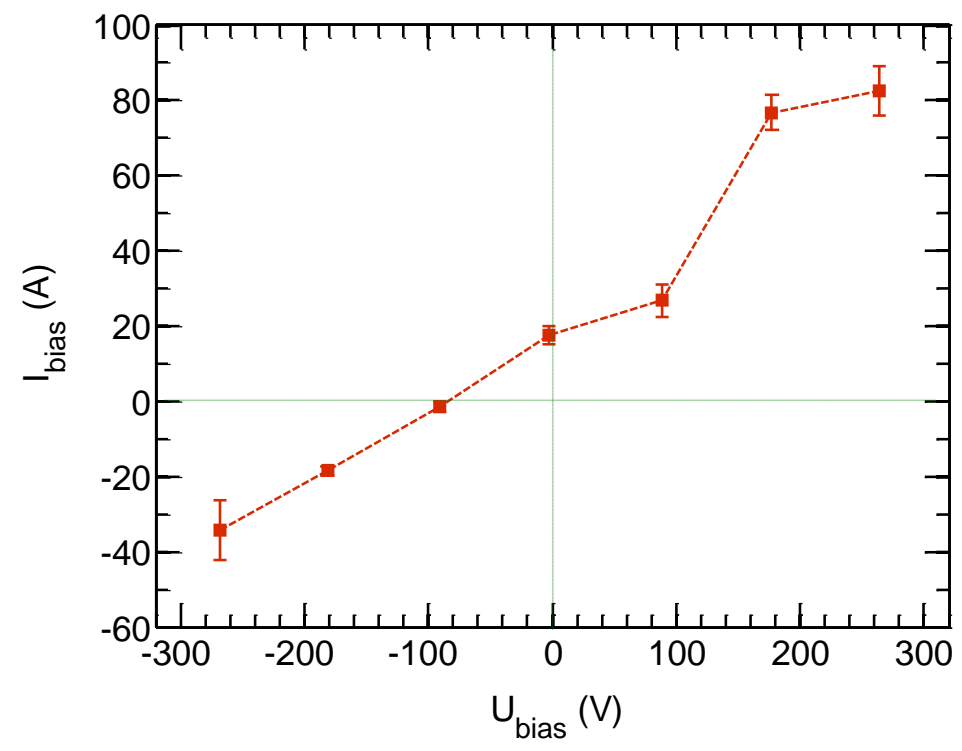

Figure 2. Volt-ampere characteristic of biased electrode

\section{Data analysis methods}

\subsection{Correlation analysis and turbulent particle flux}

Correlation analysis method is one of the most commonly used techniques in fluctuation study to estimate the coherence and cross-phase between two arbitrary stationary signals. For two given time series $x(t)$ and $y(t)$, the spectra of coherence and cross-phase $\gamma_{\mathrm{xy}}(f)$ and cross-phase $\theta_{\mathrm{xy}}(f)$ are defined as: 


$$
\begin{aligned}
& \gamma_{x y}(f)=\frac{\left|P_{x y}(f)\right|}{\sqrt{P_{x}(f) \cdot P_{y}(f)}}, \\
& \theta_{x y}(f)=\operatorname{angle}\left(P_{x y}(f)\right)=\tan ^{-1}\left(\frac{\operatorname{Im}\left(P_{x y}(f)\right)}{\operatorname{Re}\left(P_{x y}(f)\right)}\right),
\end{aligned}
$$

where $P_{\mathrm{x}}(f)$ and $P_{\mathrm{y}}(f)$ are the corresponding power spectra of signals $x(t)$ and $y(t)$, and $P_{\mathrm{xy}}(f)$ is their cross-power spectrum [31]. Through the coherence and cross-phase spectra, one can obtain the distribution of correlation between two signals as a function of frequency. The averaged coherence $\gamma_{\mathrm{xy}}$ and phase-shift $\theta_{\mathrm{xy}}$ can be evaluated as:

$$
\begin{aligned}
\gamma_{x y} & =\frac{\int\left|P_{x y}(f)\right| d f}{\sqrt{\int P_{x}(f) d f \cdot \int P_{y}(f) d f}}, \\
\theta_{x y} & =\frac{\int\left|P_{x y}(f)\right| \cdot \theta_{x y}(f) d f}{\int\left|P_{x y}(f)\right| d f} .
\end{aligned}
$$

The particle flux $\Gamma_{\mathrm{r}}$ driven by the fluctuations of density $n_{\mathrm{e}}$ and radial velocity $V_{\mathrm{r}}$ is defined as:

$$
\Gamma_{r}=<\tilde{n}_{e} \cdot \tilde{V}_{r}>=<\tilde{n}_{e} \cdot \tilde{E}_{\theta}>/ B_{t} .
$$

The influence of electron temperature $T_{\mathrm{e}}$ fluctuation is ignored in our analysis, so the density fluctuation can be substituted by the fluctuation of ion saturation current $I_{\mathrm{s}}$, and the turbulent particle flux can be estimated as:

$$
\Gamma_{r} \approx<\tilde{I}_{s} \cdot \tilde{E}_{\theta}>/ B_{t}=\sqrt{<\tilde{I}_{s}^{2}>\cdot<\tilde{E}_{\theta}^{2}>} \cdot \gamma_{I E} \cdot \cos \left(\theta_{I E}\right) / B_{t} .
$$

That is, the turbulence-driving particle transport is determined by the fluctuation levels (i.e. root-mean-square values) of ion saturation current $I_{\mathrm{s}}$ and poloidal electric field $E_{\theta}$, as well as their coherence $\gamma_{\mathrm{IE}}$ and cross-phase $\theta_{\mathrm{IE}}$. The latter two terms can be calculated by Eqs. (4) and (5) respectively.

\subsection{Conditional average}

The meso-scale, intermittent events (i.e. blobs), which can contribute a significant portion of the turbulent transport at plasma-edge, are well embedded in the ambient turbulence. Conditional average is a useful method to separate the events, as well as their related parameters, from the ambient turbulence for detailed study [32]. By presetting a certain level of threshold, usually several times of standard deviation, those succeed events which satisfy the condition are defined and orientated in the conditional signal. Then conditional average is obtained by windowing the target signal into slices at the corresponding time of events, which are accumulated and averaged finally as the result. If the conditional signal and the target 
signal are the same, it is called an auto-conditional average (ACA). Otherwise, the method is called cross-conditional average (CCA). In our analysis, ion saturation current $I_{\mathrm{s}}$ is set as the conditional signal, and the preset threshold is 2.5 times of standard deviation of $I_{\mathrm{s}}$ fluctuation. Information of the blobs, including the amplitude, radial velocity and related particle flux are obtained and analyzed with the help of ACA and CCA, as presented in the section 4.2.5.

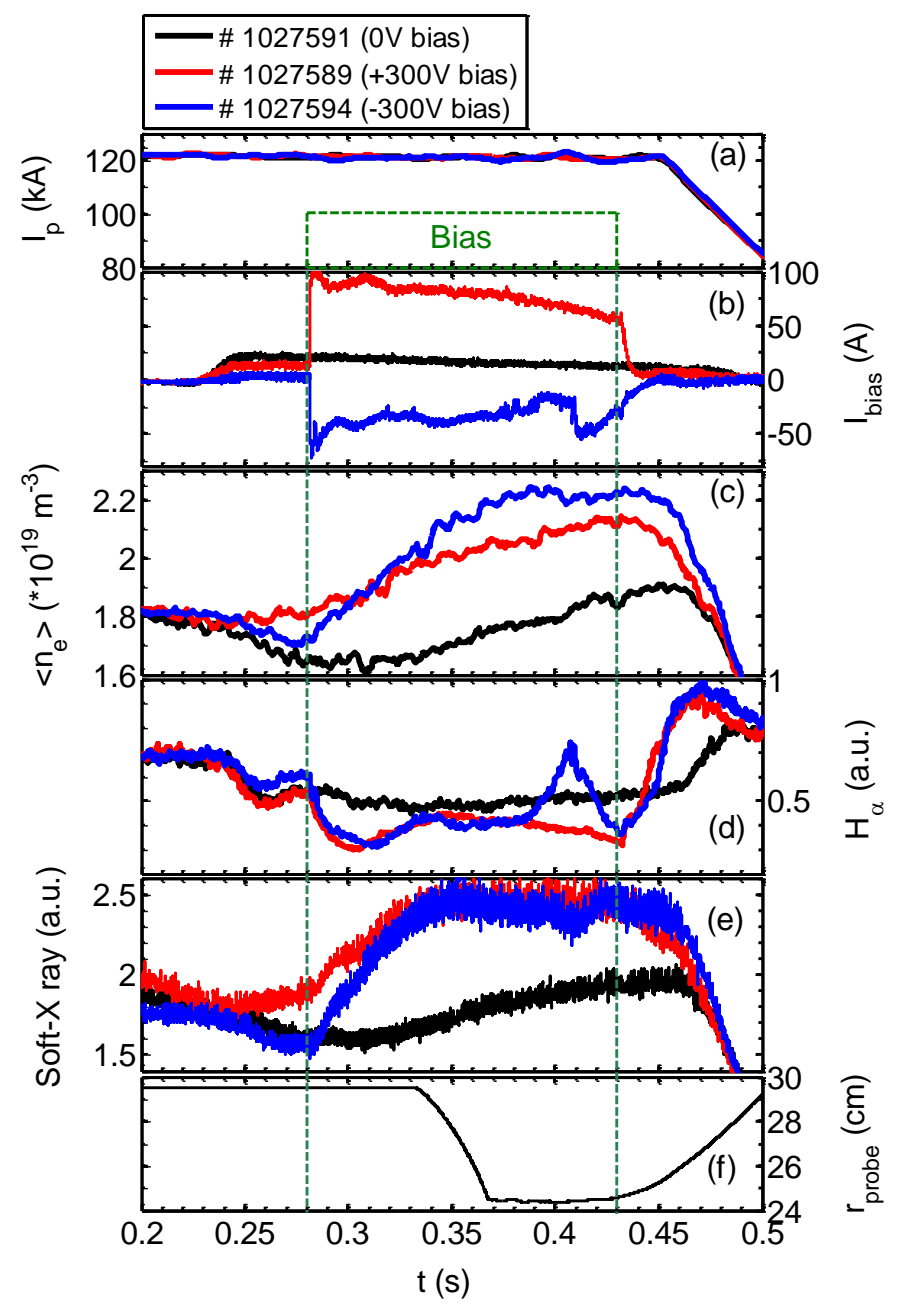

Figure 3. Evolutions of (a) plasma current, (b) electrode current, (c) central line-averaged density, (d) edge $\mathrm{H}_{\alpha}$ radiation, (e) central line-averaged soft-X ray emission and (f) trajectory of Langmuir probe under different bias cases. The interval of bias is indicated.

\section{Experimental results}

\subsection{Influence on global confinement}

To compare the influences on plasma behavior with different bias conditions, three typical discharges are selected, including shot \# 1027591 with $0 \mathrm{~V}$ bias, shot \# 1027589 with positive $(+300 \mathrm{~V})$ bias and shot \# 1027594 with negative $(-300 \mathrm{~V})$ bias. For convenience, the description of positive (negative) bias represents the $+300 \mathrm{~V}(-300 \mathrm{~V})$ bias case hereafter in this 
paper, unless otherwise noted. The evolutions of plasma parameters of these discharges are presented with three distinct colors of black, red and blue in figure 3. In these discharges, the electrode was inserted to the position $r=22.5 \mathrm{~cm}$ (i.e. $4 \mathrm{~cm}$ inside limiter) at about $0.25 \mathrm{~s}$, with a bias duration of $150 \mathrm{~ms}$ in the interval $0.28 \sim 0.43 \mathrm{~s}$. In the bias phase, the electrode current $I_{\text {bias }}$ drawn under $+300 \mathrm{~V}$ bias is in the range $60 \sim 100 \mathrm{~A}$, with a slow decrease with time, which is larger than the $-300 \mathrm{~V}$ bias case where $I_{\text {bias }}=20 \sim 70$ A. Comparing to the case of positive bias, the variation of plasma state is inferred to be relatively greater under negative bias, with a greater change of electrode current as well as plasma parameters, including plasma density and edge $\mathrm{H}_{\alpha}$ level. The global particle confinement is improved under both polarity bias cases. In the positive bias case, the overall increments of central line-averaged density and soft-X ray emission level are about $18 \%$ and $30 \%$ respectively, while the edge $\mathrm{H}_{\alpha}$ radiation level decreases by a factor of about 1.7. In the negative bias case, the evolution of plasma state is more complicated, with a degradation of particle confinement occurring at around $0.4 \mathrm{~s}$, which is evidenced by the obvious increase of edge $\mathrm{H}_{\alpha}$ level and the electrode current amplitude without increase of central line-averaged density. The overall increments of central line-averaged density and soft-X ray in the negative bias case are about $30 \%$ and $58 \%$, respectively, while the edge $\mathrm{H}_{\alpha}$ radiation level decreases by a factor of about 1.9. The trace of the reciprocating Langmuir probe is shown in figure 3 (f). The profiles provided below are obtained from the probe data during the inserting phase $(0.33 \sim 0.37 \mathrm{~s})$.
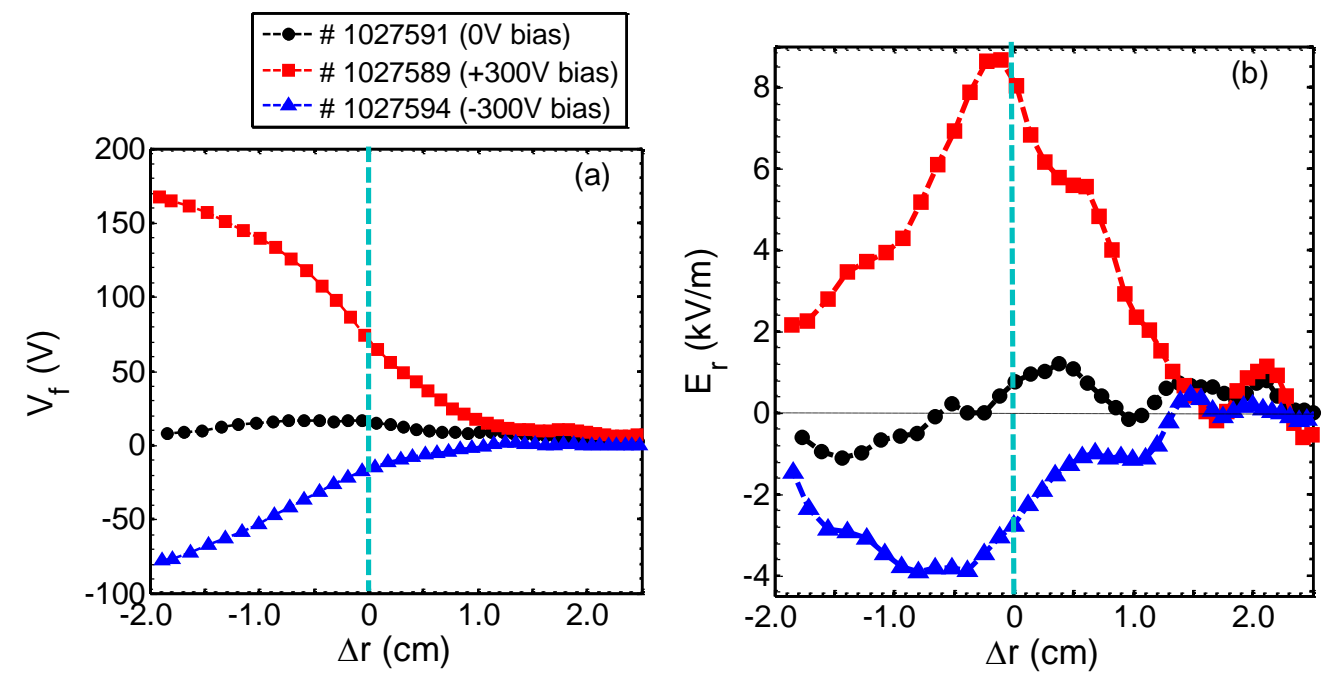

Figure 4. Equilibrium profiles of (a) floating potential $V_{\mathrm{f}}$ and (b) radial electric field $E_{\mathrm{r}}$ under different bias cases at plasma-edge. The vertical lines indicate the position of limiter.

\subsection{Influence on plasma-edge parameters}

4.2.1. Equilibrium and fluctuation profiles. The plasma-edge equilibrium profiles of floating potential $V_{\mathrm{f}}$ and radial electric field $E_{\mathrm{r}}$ are obtained under different bias conditions and presented in figure 4 . The horizontal coordinates of the profiles in this paper are indicated as 
the difference between the radial position of reciprocating Langmuir probe $r_{\text {probe }}$ and the minor radius $a$, i.e. $\Delta r=r_{\text {probe }}-a$. Thus, $\Delta r=0$ represents the position of limiter (or last closed flux surface), while $\Delta r<0$ represents the plasma-edge region inside limiter. The profiles of $V_{\mathrm{f}}$ are modified greatly according to the polarities of bias at edge inside limiter. Ignoring the contribution from electron temperature, the radial electric field $E_{\mathrm{r}}$ can be deduced from the profile of floating potential $V_{\mathrm{f}}$ by:

$$
E_{r}=-d V_{f} / d r
$$

Here, the positive $E_{\mathrm{r}}$ indicates a radially outside electric field. Comparing to the $0 \mathrm{~V}$ bias case, both the absolute value and shearing rate of $E_{\mathrm{r}}$ are obviously increased under bias with both polarities. Under positive bias, $E_{\mathrm{r}}$ reaches a maximum value about $8.6 \mathrm{kV} / \mathrm{m}$ at $\Delta r=-0.2 \mathrm{~cm}$. The averaged shearing rate of $E \times B$ velocity $\gamma_{\mathrm{E \times B}}$ in the broad shear layer $(\Delta r=-2.0 \sim 1.5 \mathrm{~cm})$ is about $2.4 \times 10^{5} \mathrm{~s}^{-1}$, which is calculated as

$$
\gamma_{\vec{E} \times \bar{B}}=\left|\frac{d V_{\vec{E} \times \vec{B}}}{d r}\right|=\left|\frac{d E_{r}}{B_{t} \cdot d r}\right| .
$$

While under negative bias, $E_{\mathrm{r}}$ reaches a minimum value about $-4 \mathrm{kV} / \mathrm{m}$ at $\Delta r=-0.5 \mathrm{~cm}$, with the mean shearing rate in the shear layer $(\Delta r=-2.0 \sim 1.5 \mathrm{~cm})$ as $1.1 \times 10^{5} \mathrm{~s}^{-1}$. Comparatively, the shearing rate for the $0 \mathrm{~V}$ bias case is as weak as about $0.5 \times 10^{5} \mathrm{~s}^{-1}$ at the vicinity of limiter.

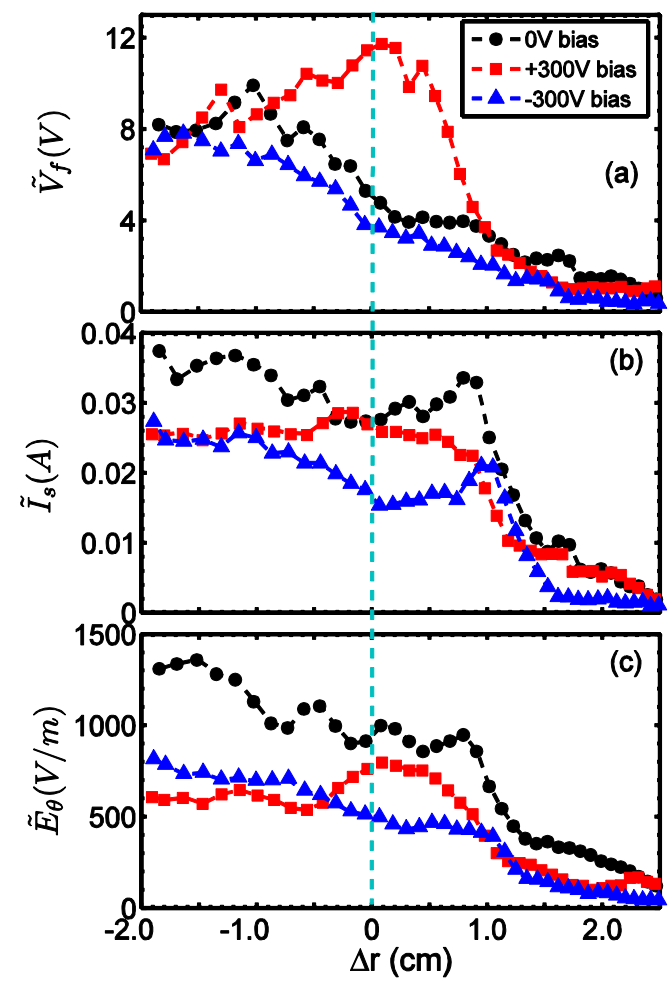

Figure 5. Profiles of fluctuations for (a) floating potential $V_{\mathrm{f}}$, (b) ion saturation current $I_{\mathrm{s}}$ and (c) poloidal electric field $E_{\theta}$ under different bias cases. The vertical line indicates the position of limiter. 
The modification of the fluctuations of plasma-edge parameters is found to be sensitive to the polarities of bias, especially for the floating potential $V_{\mathrm{f}}$, as shown in figure 5 . Under positive bias, the amplitude of $V_{\mathrm{f}}$ fluctuation is increased at the range $\Delta r=-0.8 \sim 1.0 \mathrm{~cm}$, which is mainly due to the growth of the low-frequency power below $50 \mathrm{kHz}$, as revealed in the corresponding space-resolved power spectrum (see figure 6 (c)) based on the short-time Fourier transform (STFT) technique. While for the negative bias case, the fluctuation of $V_{\mathrm{f}}$ is suppressed in the whole edge region, which is consistent with an obvious reduction of the spectral power density in the low-frequency range $(<20 \mathrm{kHz})$, as shown in figure $6(a)$. The fluctuation levels of $I_{\mathrm{s}}$ and poloidal electric field $E_{\theta}$ are all decreased under bias with both polarities, but in different degrees. The suppression of these fluctuation quantities is less effective in the range $\Delta r=-0.5 \sim 1.0 \mathrm{~cm}$ under positive bias (see figure 5 (b) and (c)), with a broadening of the corresponding frequency spectra, which is not clear observed in the case of negative bias (see figure 6 (d) (i)).
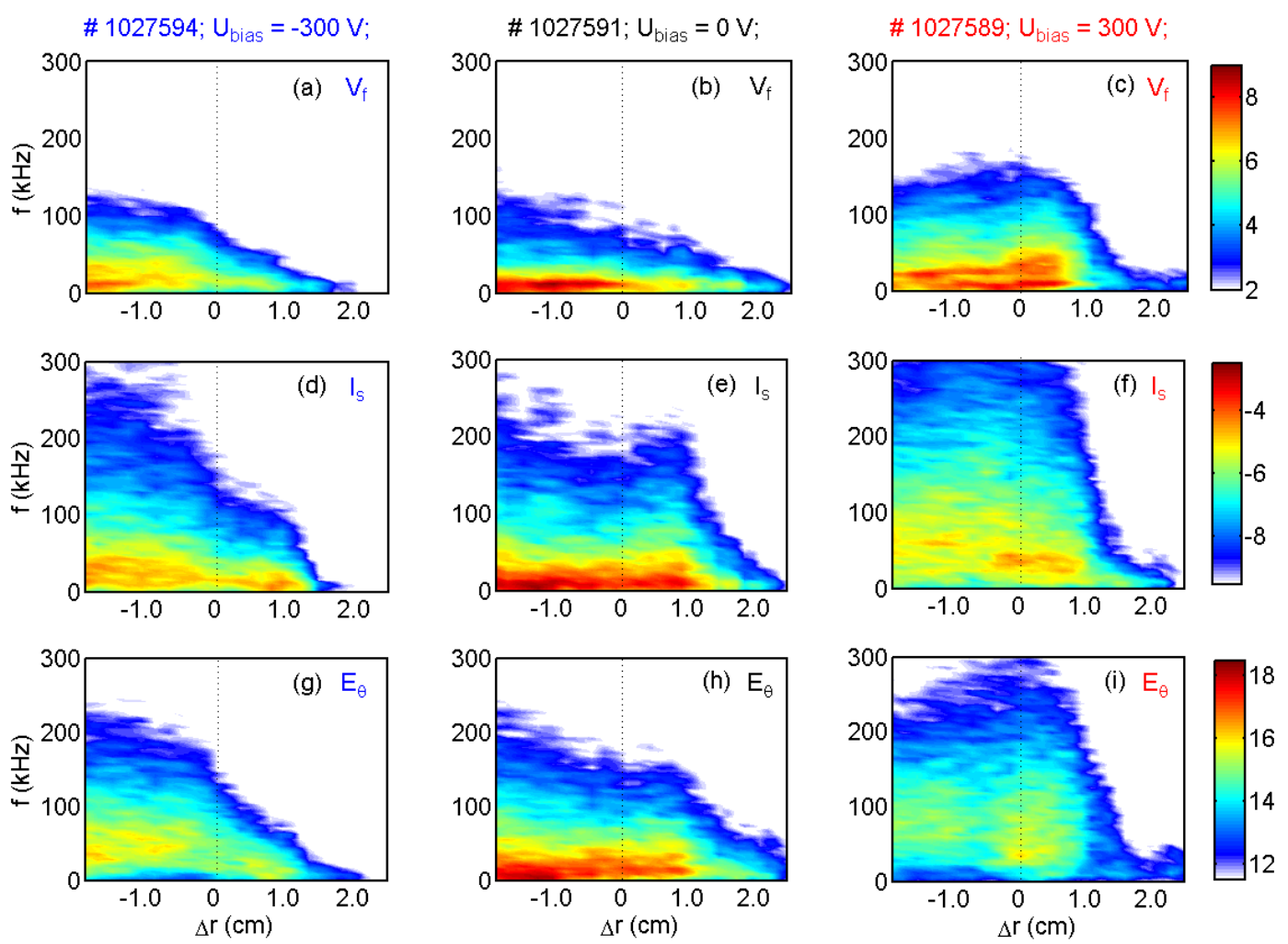

Figure 6. Space-resolved power spectra of $I_{\mathrm{s}}((\mathrm{a}) \sim(\mathrm{c})), V_{\mathrm{f}}((\mathrm{d}) \sim(\mathrm{f}))$ and $E_{\theta}((\mathrm{g}) \sim(\mathrm{i}))$ based on the short-time Fourier transform (STFT) technique, under different bias cases. Spectra of each parameter correspond to a unified color bar which denotes the logarithm of power density. The vertical lines indicate the position of limiter.

4.2.2. Turbulent particle flux. As previously introduced, the turbulence-driving particle flux, $\Gamma_{\mathrm{r}}$, is determined by the fluctuation levels of $I_{\mathrm{s}}$ and $E_{\theta}$, as well as their coherence $\gamma_{\mathrm{IE}}$ and cross-phase $\theta_{\mathrm{IE}}$, according to Eq. (7) in section 3.1, under the assumption of ignoring the 
influence of electron temperature fluctuation. The fluctuation levels of $I_{\mathrm{s}}$ and $E_{\theta}$, as have been provided and discussed above, are suppressed in the whole edge region, but in different degrees for different biased polarities. At the vicinity of limiter $(\Delta r=-0.5 \sim 1.0 \mathrm{~cm})$, the extent of suppression on fluctuations of $I_{\mathrm{s}}$ and $E_{\theta}$ under positive bias is weaker compared to the negative case. Moreover, their coherent and cross-phase spectra, as presented in figure 7 and figure 8 , also behave differently according to the polarities. Under positive bias, the coherence $\gamma_{\mathrm{IE}}$ is even strengthened inside limiter, which is due to the enhancement of coherence in a broad-frequency range, as shown in figure 8 (c). Their cross-phase $\theta_{\mathrm{IE}}$ is kept small where the coherence is high, making the suppression on fluctuation levels as the only contributor to the reduction of turbulent particle flux in the edge under positive bias. In negative bias case, the coherence $\gamma_{\mathrm{IE}}$ is decreased at the vicinity of limiter, while the cross-phase related term $\cos \left(\theta_{\mathrm{IE}}\right)$ is modulated greatly inside the limiter, which decreases to be negative at $\Delta r \sim-2.0 \mathrm{~cm}$ (see figure 7 (b) and figure 8 (d)). Thus, the reduction of turbulent particle flux under negative bias is attributed to the combined role of fluctuation suppression and de-phasing at the edge region that inside limiter. The degree of suppression on turbulent particle flux is relatively greater under negative bias, compared to the positive bias case (see figure 7 (c)).

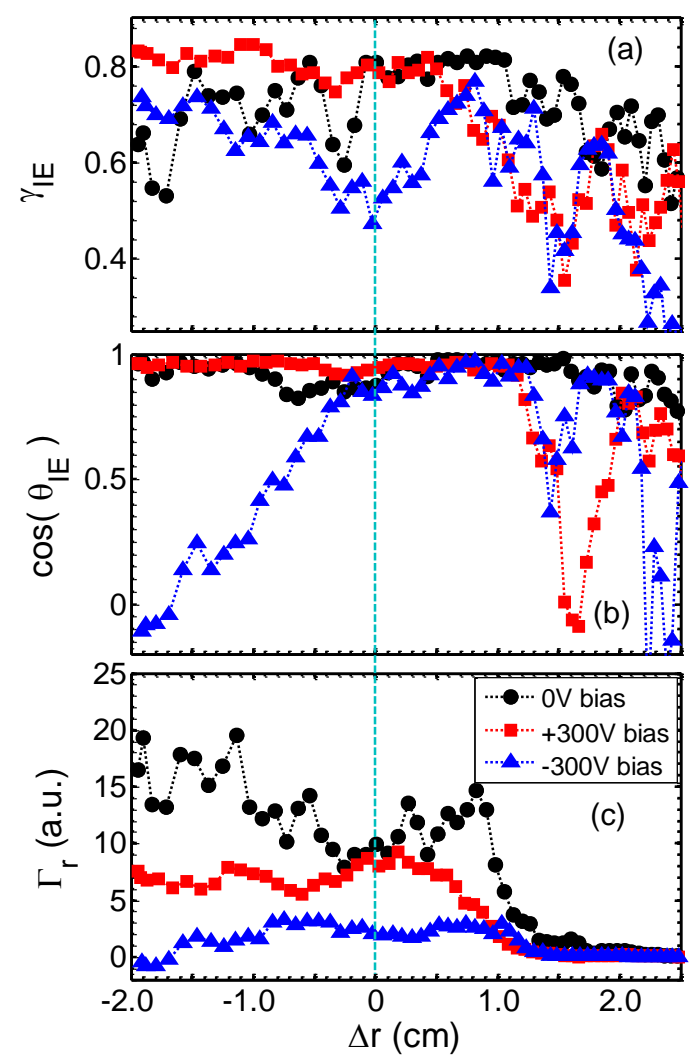

Figure 7. Profiles of coherence (a), cross-phase related term $\cos \left(\theta_{\mathrm{IE}}\right)(\mathrm{b})$ and resulting turbulent particle flux (c) under different bias cases. The vertical line indicates the position of limiter. 

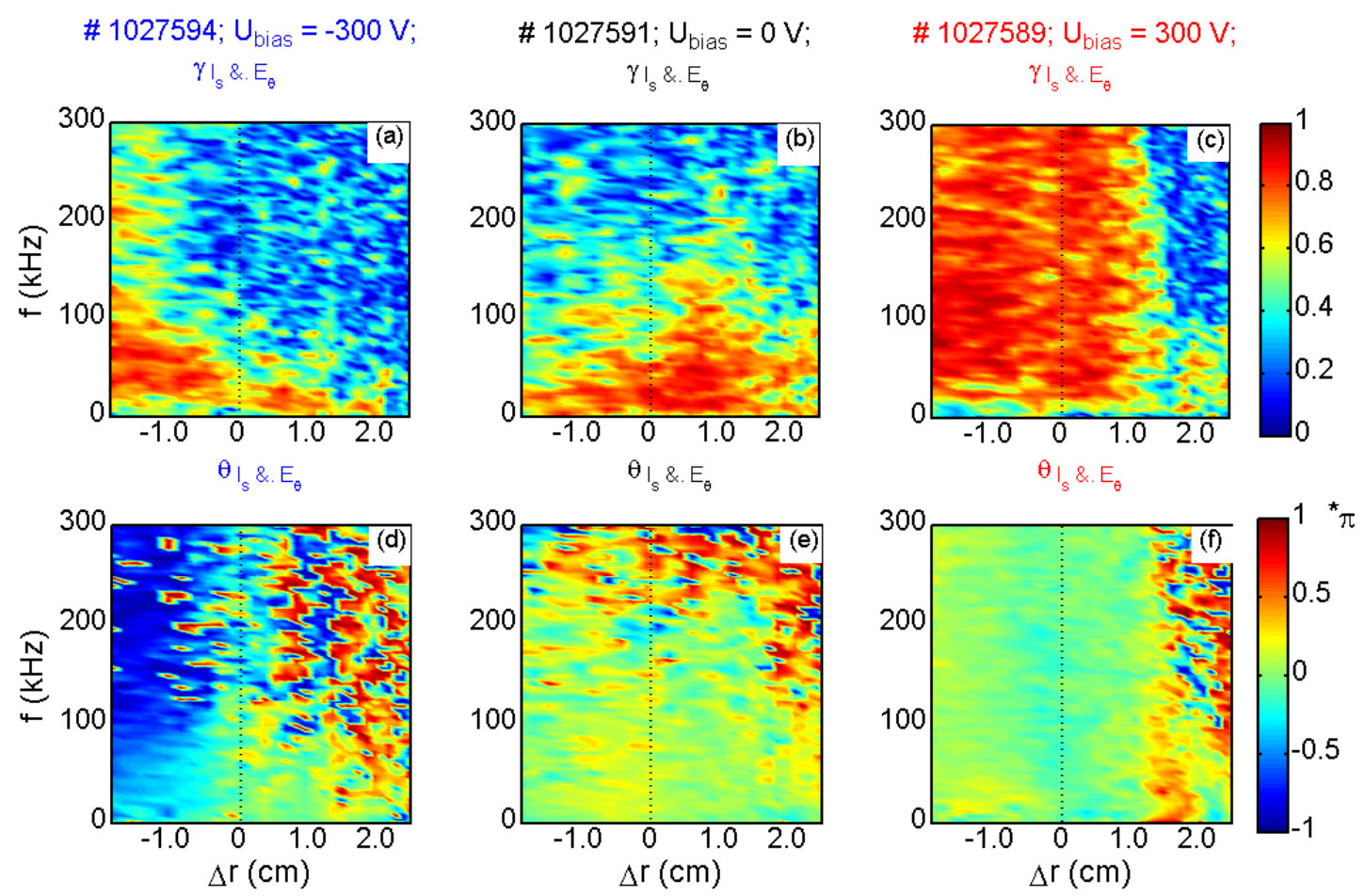

Figure 8. Space-resolved spectra of coherence $((\mathrm{a}) \sim(\mathrm{c}))$ and cross-phase $((\mathrm{d}) \sim(\mathrm{f}))$ between $I_{\mathrm{s}}$ and $E_{\theta}$ fluctuations under different bias cases. The vertical lines indicate the position of limiter.
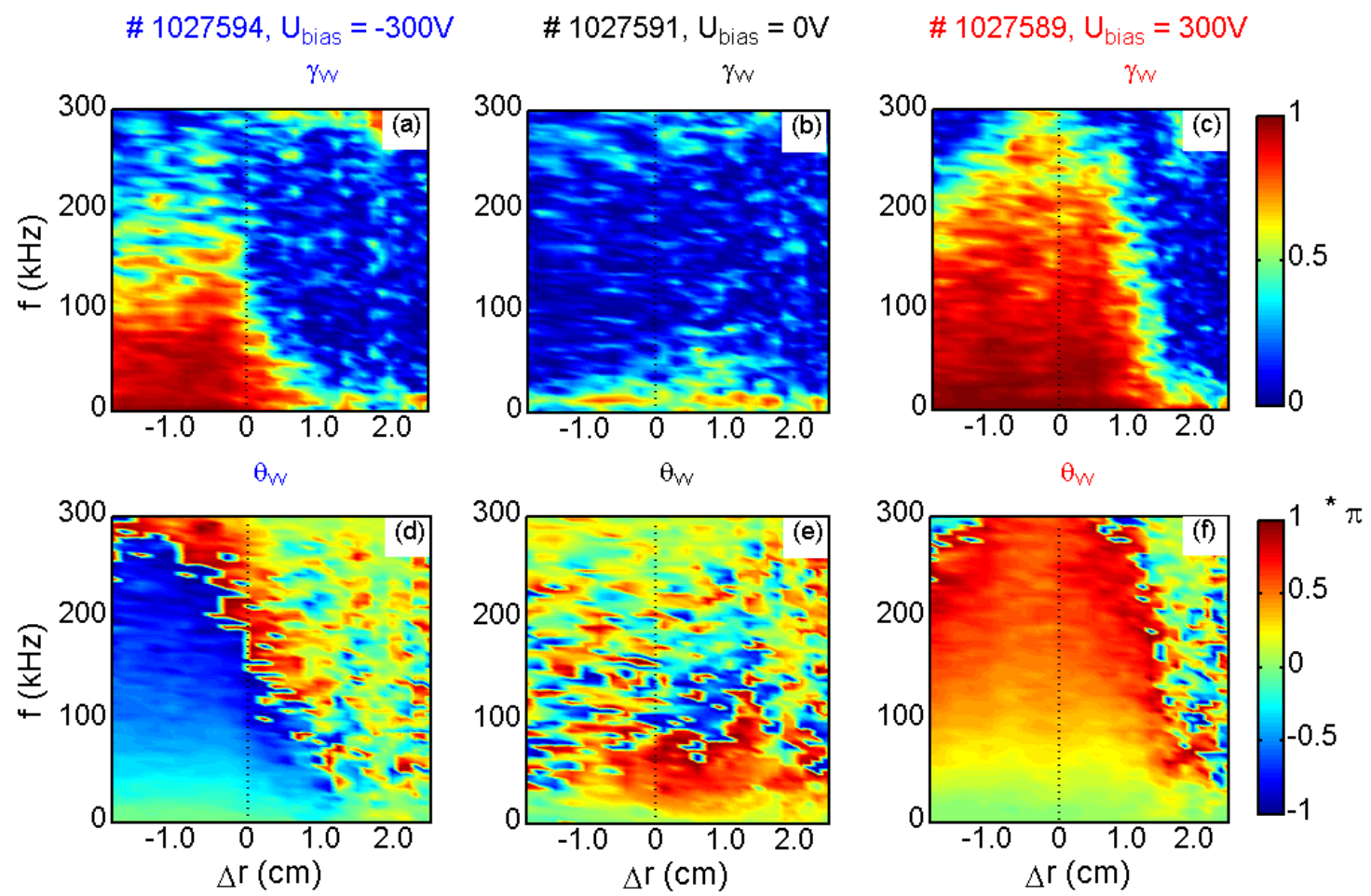

Figure 9. Space-resolved spectra of coherence $((\mathrm{a}) \sim(\mathrm{c}))$ and cross-phase $((\mathrm{d}) \sim(\mathrm{f}))$ between two floating potential fluctuations with $7 \mathrm{~mm}$ poloidal separation, under different bias cases. The vertical lines indicate the position of limiter. 


\subsubsection{Details of potential fluctuations. The coherent and cross-phase spectra between two}

floating potentials $V_{\mathrm{f} 1}$ and $V_{\mathrm{f} 2}$, which are poloidally separated by about $7 \mathrm{~mm}$, are provided in figure 9. Comparing to the $0 \mathrm{~V}$ bias case, the coherence distribution as a function of frequency are much higher in a broad range under bias with both polarities, as shown in figure 9 ((a) (c)). And the high-coherence region under positive bias is relatively broader in both space and frequency domain than the negative bias case. The cross-phase keeps varying slowly in an approximate linear way in the region where the coherence is high (see figure 9 ((d) (f))). This high-coherence behavior of the potential fluctuations could be related to the enhancement of poloidal velocity of local plasma, as will be discussed later.

To further investigate the unrevealed property of turbulence, the local wavenumber-frequency spectrum is estimated based on the two-point spectral analysis technique [28], in which the fluctuations of the two poloidally separated floating potentials, $V_{\mathrm{f} 1}$ and $V_{\mathrm{f} 2}$, are employed as the data source. Figure 10 shows the resulting wavenumber-frequency spectra $S\left(k_{\theta}, f\right)$ obtained at $\Delta r=-2 \mathrm{~cm}$ under different bias conditions. For $0 \mathrm{~V}$ bias case, the spectral power density is mainly located in the low-frequency range that is below $30 \mathrm{kHz}$, with a broad distribution of poloidal wavenumber $k_{\theta}$, as shown in figure 10 (b). Here, the positive $k_{\theta}$ indicates the ion diamagnetic drift (IDD) direction, while the negative one indicates the electron diamagnetic drift (EDD) direction. Under negative bias, the spectral power density mainly locates in the negative $k_{\theta}$ region, and concentrates in a narrow space of low-frequency $(<100 \mathrm{kHz})$ and small $k_{\theta}\left(-2 \sim 0 \mathrm{~cm}^{-1}\right)$, as presented in figure 10 (a). The related dispersion relation shows a quasi-linear behavior in the ranges $0 \sim 30$ and $30 \sim 100 \mathrm{kHz}$ respectively, with different slopes. The poloidal phase velocity of turbulence $V_{\text {ph }}$ estimated by the two-point spectral analysis technique is about $2 \mathrm{~km} / \mathrm{s}$ towards EDD direction. Under positive bias, the distribution of spectral power density shows a clear turning point at around $15 \mathrm{kHz}$, as marked in figure 10 (c). In the frequency range below $15 \mathrm{kHz}$, the wavenumber $k_{\theta}$ keeps close to $0 \mathrm{~cm}^{-1}$, with the power density peaks at $f \sim 12 \mathrm{kHz}$. While in the frequency range above $15 \mathrm{kHz}$, the spectral power density locates in the positive wavenumber region in an approximate linear way, with the power density peaks at $f \sim 21 \mathrm{kHz}$. The phase velocity estimated is about $4 \mathrm{~km} / \mathrm{s}$ towards IDD direction. There are two low-frequency modes (LFMs) exist under positive bias at this position $(\Delta r=-2 \mathrm{~cm})$, with the peaked frequencies as $12 \mathrm{kHz}$ and $21 \mathrm{kHz}$ respectively, as shown in the cross-spectra in figure 11. The $12 \mathrm{kHz}$ LFM also exists under OV bias case but is absent under negative bias case. This mode may correspond to a geodesic acoustic mode (GAM) with extremely high coherence and near-zero cross-phase between floating potential fluctuations with $7 \mathrm{~mm}$ poloidal separation. A theoretical prediction of GAM frequency by $f_{G A M}^{\text {theo. }}=C_{s} /(2 \pi R)$ [33] is $11.7 \mathrm{kHz}$ with the relevant parameters on J-TEXT (major radius $\mathrm{R}=1.05 \mathrm{~m}$, and local 
temperatures $\left.T_{\mathrm{i}} \sim T_{\mathrm{e}} \sim 30 \mathrm{eV}\right)$, and this predicted frequency is rather close to the observed frequency of the LFM ( $\sim 12 \mathrm{kHz})$. The other LFM under positive bias $(f \sim 21 \mathrm{kHz})$ also shows a high-coherence behavior, with a wavenumber $k_{\theta} \sim 0.2 \mathrm{~cm}^{-1}$.
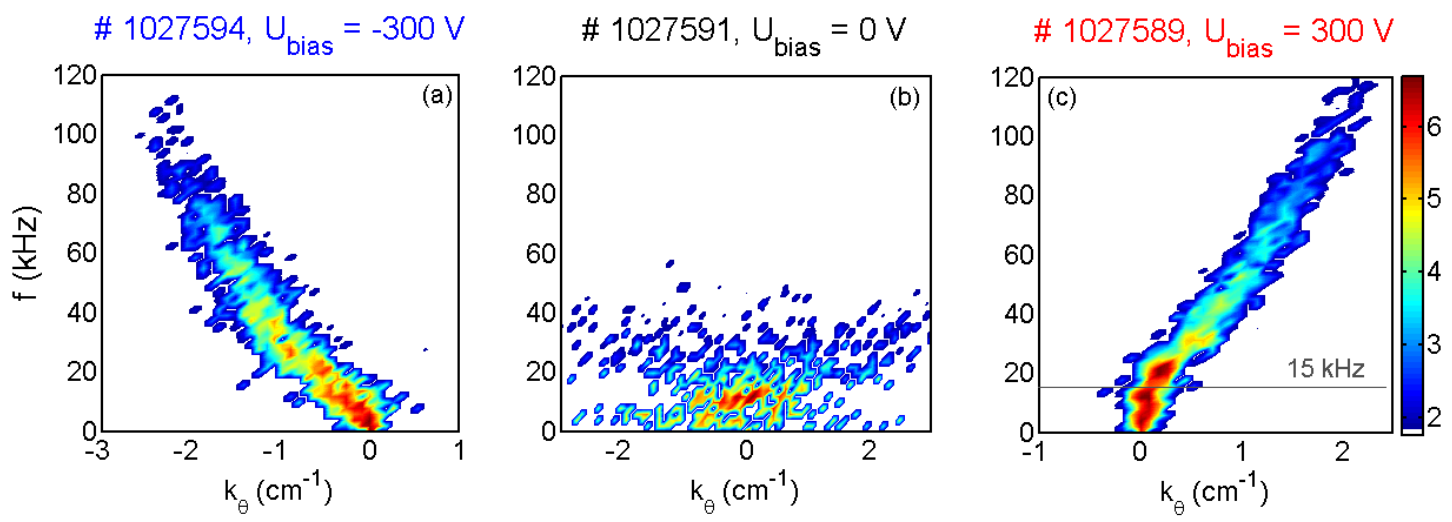

Figure 10. Frequency-wavenumber spectra $S\left(k_{\theta}, f\right)$ estimated with two poloidally separated floating potentials at $\Delta r=-2 \mathrm{~cm}$, under different bias cases.

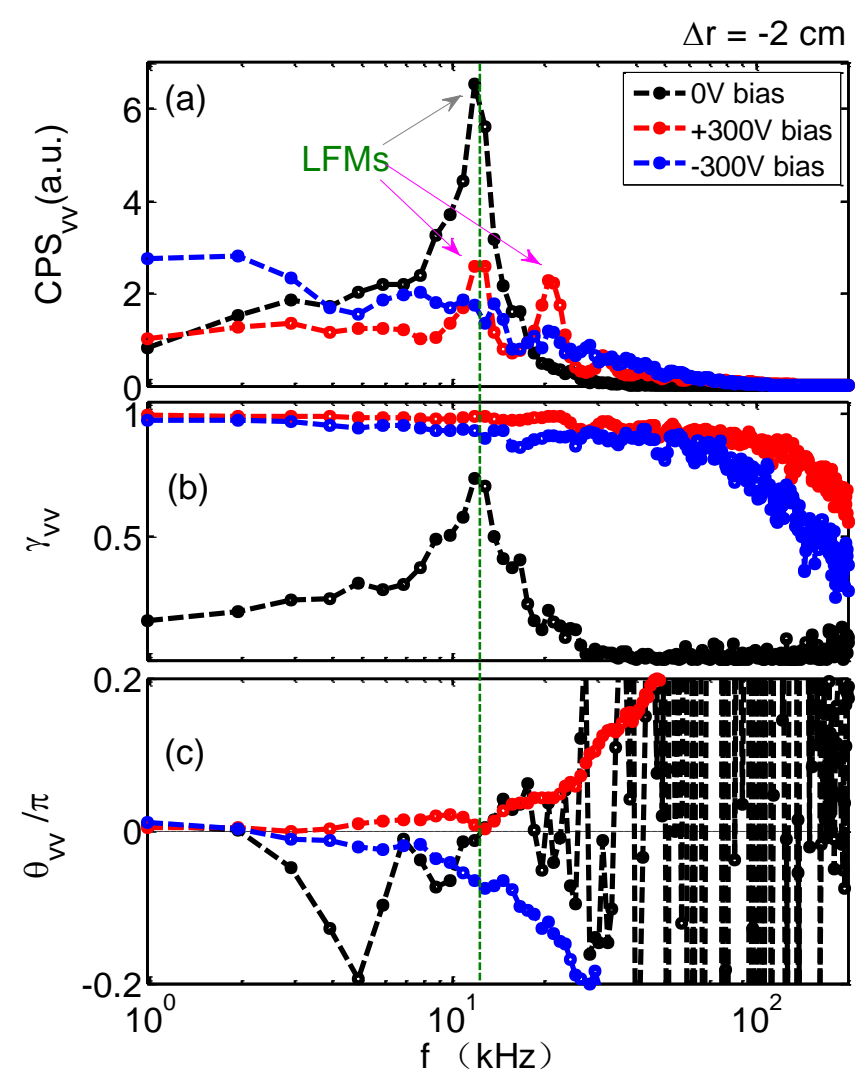

Figure 11. Cross-power spectra (CPS) (a), as well as coherence (b) and cross-phase (c) spectra between two poloidally separated floating potentials at $\Delta r=-2 \mathrm{~cm}$, under different bias conditions. The low-frequency modes (LFMs) are denoted in the spectra.

The common feature shared by the bias cases with different polarities is the tendency of broadening of frequency space as well as narrowing of the wavenumber spectral width, comparing with the 0V bias case. According to the reference [29], the narrowing of poloidal wavenumber spectral width corresponds to the enhancement of poloidal coherence of 
turbulence. The profiles of poloidal correlation length $L_{\mathrm{c} \theta}$ estimated by the two-point spectral analysis technique are given in figure 12. While the distribution of $L_{\mathrm{c} \theta}$ under $0 \mathrm{~V}$ bias is almost flat and stays in the range $1 \sim 2 \mathrm{~cm}$ in the whole measured region at plasma-edge, the amplitude of correlation length increases greatly under bias, and reaches to the level of $\sim 8 \mathrm{~cm}$ and $\sim 10 \mathrm{~cm}$ for negative and positive bias cases, repectively. The high- $L_{\mathrm{c} \theta}$ region under positive bias $(\Delta r=-2.0 \sim 1.0 \mathrm{~cm})$ is relatively broader than the nagetive bias case $(\Delta r=-2.0 \sim$ $0 \mathrm{~cm})$. Comparing to the $0 \mathrm{~V}$ bias case, the poloidal correlation length $L_{\mathrm{c} \theta}$ at plasma edge $(\Delta r=$ $-2 \mathrm{~cm}$ ) is enhanced in a wide frequency region under bias (see figure 13), which is consistent with the high-coherence behavior shown in figure 9. In addition, the LFMs contribute essential roles to the large $L_{\mathrm{c} \theta}$ in the low frequency region ( $\mathrm{f}<25 \mathrm{kHz}$ ) under $0 \mathrm{~V}$ and positive bias cases.

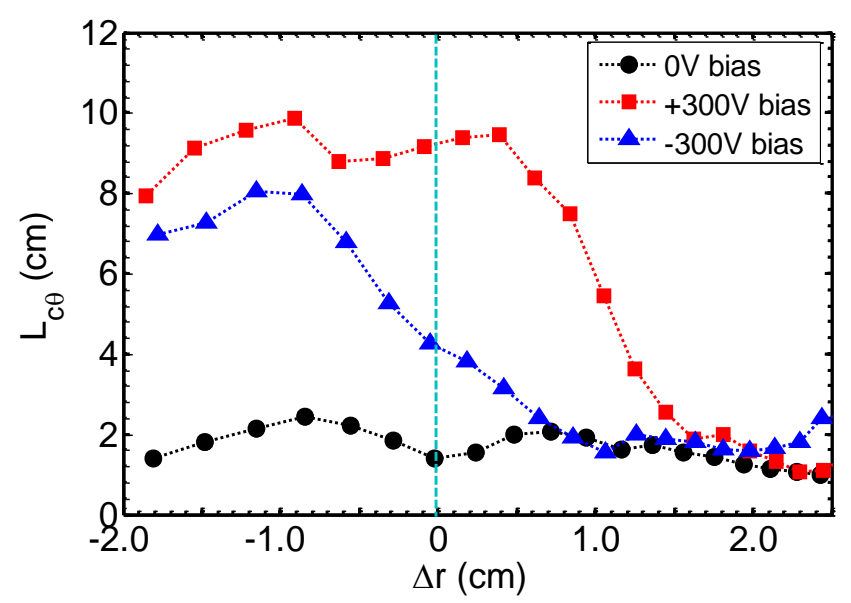

Figure 12. Profiles of poloidal correlation length of turbulence $L_{c \theta}$ under different bias cases. The vertical line indicates the position of limiter.

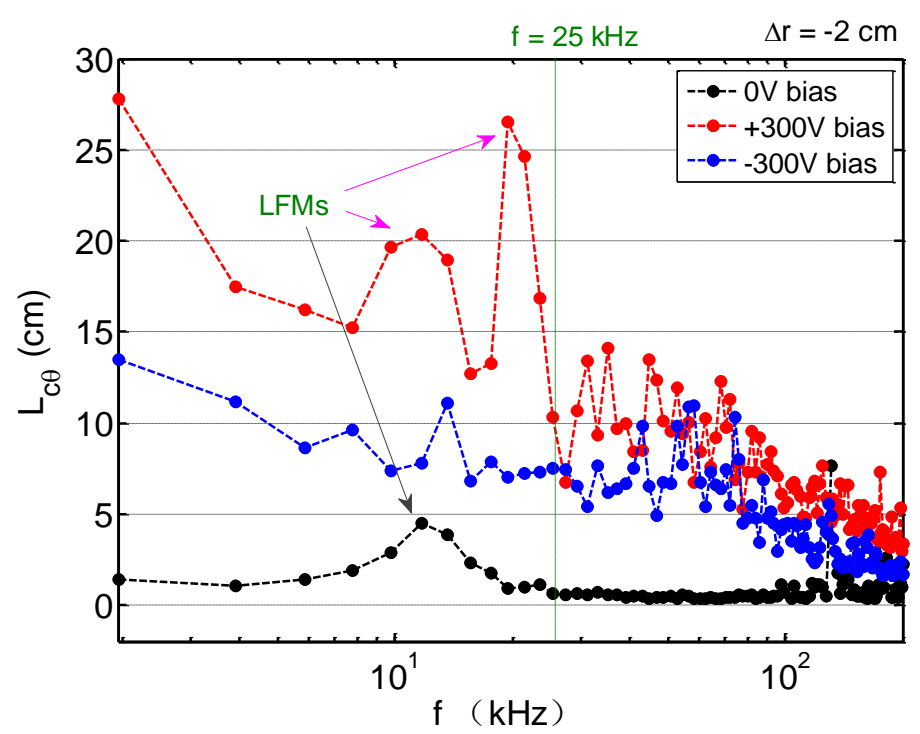

Figure 13. Frequency spectra of poloidal correlation length $L_{c \theta}$ at $\Delta \mathrm{r}=-2 \mathrm{~cm}$ under different bias cases. The low-frequency modes (LFMs) are denoted in the spectra. 
4.2.4. Poloidal velocity. The profile of poloidal phase velocity of turbulence $V_{\mathrm{ph}}$ is modified greatly under bias, as shown in figure 14 . Here, the positive value of the phase velocity indicates the IDD direction, while the negative value indicates the EDD direction. For OV bias case, the amplitude of phase velocity is low, varying slowly with radius in the range $-0.5 \sim 1$ $\mathrm{km} / \mathrm{s}$. While under positive bias, the poloidal phase velocity speeds up greatly towards IDD direction and reaches to the level of $3.5 \sim 4.5 \mathrm{~km} / \mathrm{s}$ inside limiter. Under negative bias, the phase velocity speeds up in the EDD direction, and reaches to a maximum amplitude of $\sim 2.5$ $\mathrm{km} / \mathrm{s}$ around $\Delta r=-1.5 \mathrm{~cm}$. The radial current $J_{\mathrm{r}}$ drawn by the biased electrode at plasma-edge may induce a $J_{\mathrm{r}} \times B$ torque with a significant poloidal component, and driving local plasma to speed up poloidally. This is demonstrated by the statistical result in figure 15 , which is obtained by a bias voltage scan (from $-300 \mathrm{~V}$ to $+300 \mathrm{~V}$ with the step-size as $100 \mathrm{~V}$ ) and shows a positive correlation between the poloidal phase velocity $V_{\mathrm{ph}}$ measured at $\Delta r=-2 \mathrm{~cm}$ and the radial current $J_{\mathrm{r}} . V_{\mathrm{ph}}$ has a negative value (i.e. towards EDD direction) in the absence of radial current $\left(J_{\mathrm{r}}=0\right)$, which is consistent with the existence of the spontaneous negative $E_{\mathrm{r}}$ structure just inside limiter in purely ohmic discharges on the device [27].

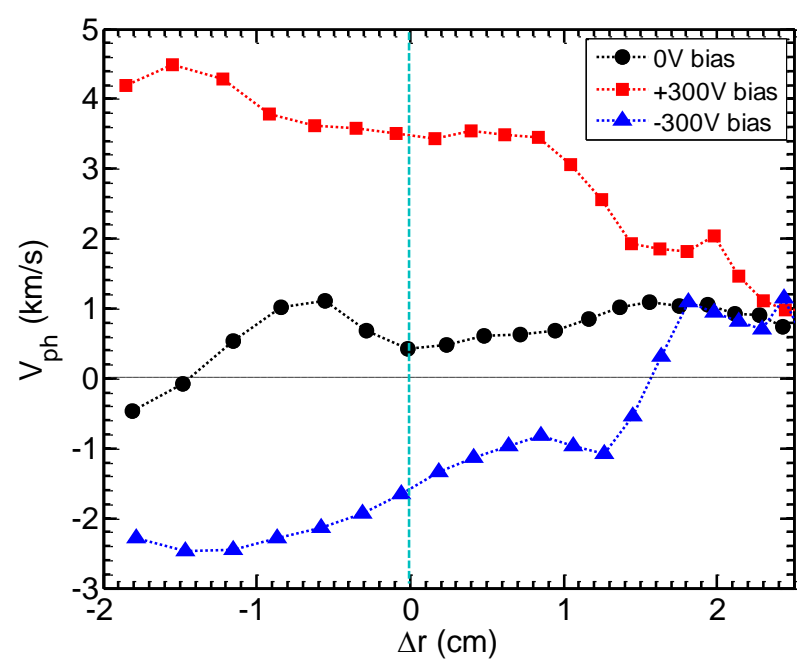

Figure 14. Profiles of poloidal phase velocity of turbulence $V_{\text {ph }}$ under different bias cases. The vertical line indicates the position of limiter. 


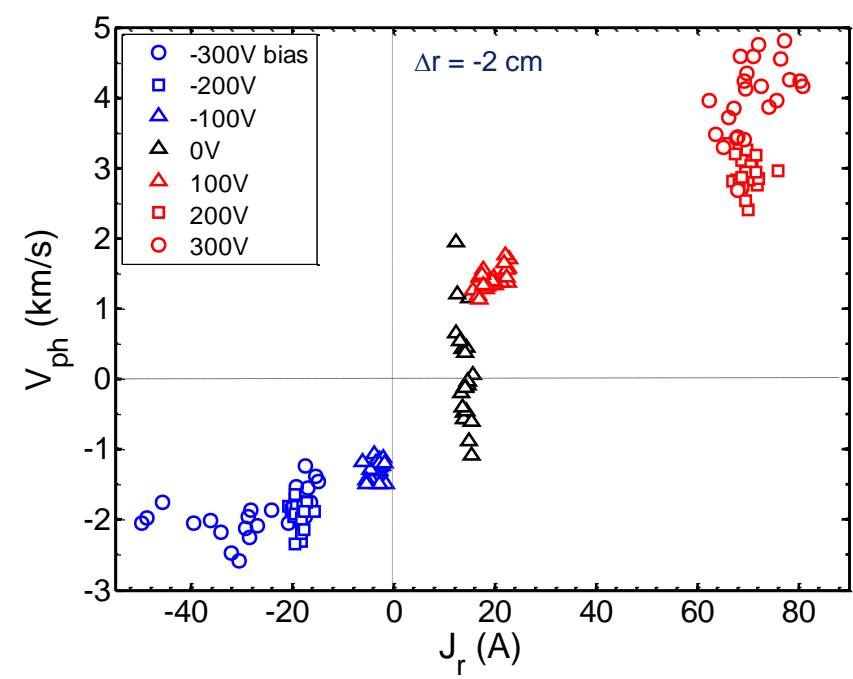

Figure 15. Distribution of $V_{\mathrm{ph}}$ measured at $\Delta r=-2 \mathrm{~cm}$ as a function of radial current $J_{\mathrm{r}}$ drawn by the biased electrode, under different bias cases (scan the bias voltage from $-300 \mathrm{~V}$ to $+300 \mathrm{~V}$, with the step-size as100V).

4.2.5. Intermittent events and related transport. The previous works show that, the intermittent events, i.e. blobs, can make a significant contribution to the total turbulent particle flux in the edge of J-TEXT tokamak with purely ohmic discharges [27, 30]. How to control the level of the events, as well as the related transport, becomes an important issue. A recent experimental work performed on a simple magnetized toroidal device, TORPEX, shows that the blobs (especially their velocities) can be controlled by the electrostatic convective cells generated by a two-dimensional array of biasing electrodes [18]. Thus, some influence of bias on the intermittent events is expected.

In our analysis, conditional average is used to separate the blobs and their related parameters, including the radial velocity and turbulent particle flux, from the ambient turbulence. Using fluctuation of ion saturation current $I_{\mathrm{s}}$ as the conditional signal and 2.5 times of its standard deviation as the threshold, the results of ACA of $I_{\mathrm{s}}$, as well as the CCAs of radial velocity $V_{\mathrm{r}}$ and turbulent particle flux $\Gamma_{\mathrm{r}}$ are shown in figure 16 . Here, the turbulent particle flux $\Gamma_{\mathrm{r}}$ is estimated by Eq. (7) and radial velocity $V_{\mathrm{r}}$ is calculated with:

$$
V_{r}=E_{\theta} / B_{t} .
$$

For the convenience of comparison, several characteristic parameters of blob are defined as follows. The blob amplitude corresponds to the difference between the maximum value and the background value, while the blob life time $\tau_{\mathrm{c}}^{\text {blob }}$ corresponds to the full width at half maximum (FWHM), of the ACA of $I_{\mathrm{s}}$ (figure 16 (a)). The blob related radial velocity $V_{\mathrm{r}}^{\text {blob }}$ and particle flux $\Gamma_{\mathrm{r}}^{\text {blob }}$ are estimated by the peak values of the corresponding CCAs (figure 16 (b) and (c)), and the radial size of blob $\delta_{\mathrm{r}}^{\text {blob }}$ is calculated by: 


$$
\delta_{r}^{b l o b}=V_{r}^{b l o b} \cdot \tau_{c}^{b l o b} .
$$

In addition, the statistical property of the intermittency of density fluctuation, which is characterized by the non-Gaussian probability distribution function (PDF), can be quantified as skewness (S) and kurtosis (K). The corresponding formulas are given by:

$$
\begin{aligned}
& S=<(x-<x>)^{3}>/ \sigma^{3}, \\
& K=<(x-<x>)^{4}>/ \sigma^{4} .
\end{aligned}
$$

Here, $\sigma$ is the standard deviation of a random, stationary signal $x$. For a Gaussian signal, the values of $S$ and $K$ are 0 and 3, respectively.

Comparing to the 0V bias case, the skewness of density fluctuation is obviously suppressed under positive bias inside limiter, where the distribution of kurtosis has little change, as shown in figure 17 (a) and (b). Together with that, the blob related parameters, including the amplitude, radial velocity and the resulting particle flux are all decreased in the same region, as shown in figure 17 (c) (e). For negative bias case, the distributions of skewness and kurtosis even show a little increase inside limiter, and the decreases of the blob characteristic parameters are mainly due to the suppression of the fluctuation levels of $I_{\mathrm{s}}$ and $E_{\theta}$. Apart from this, the out-phase between $I_{\mathrm{s}}$ and $E_{\theta}$ fluctuations could be another contributor to the reduction of blob related particle flux inside limiter.

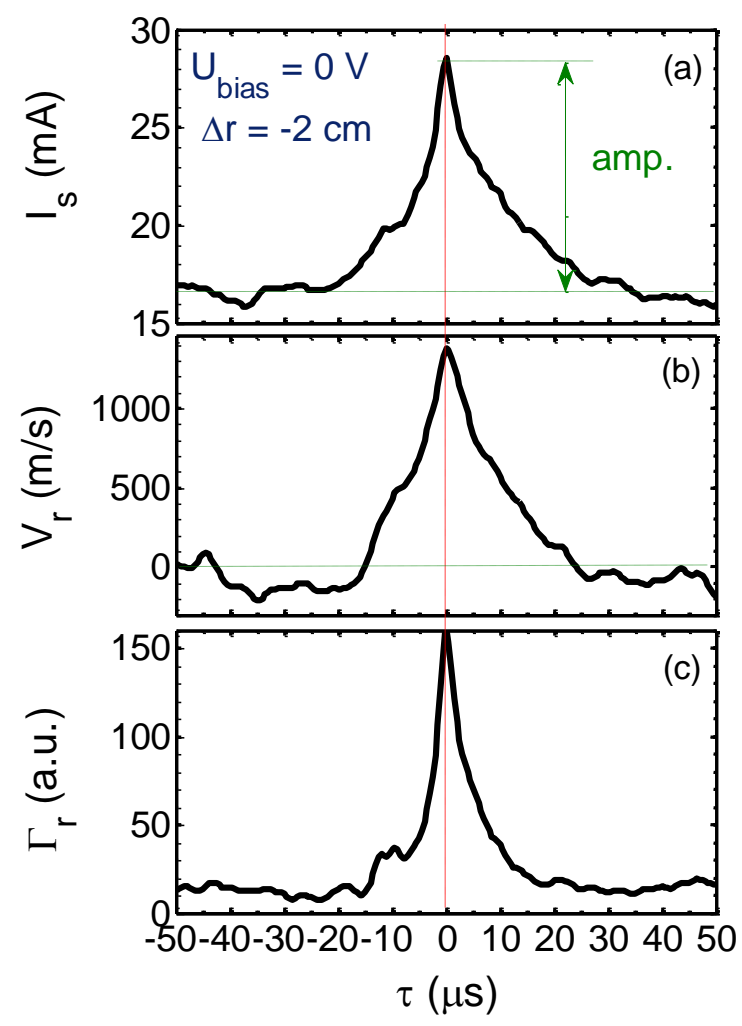

Figure 16. ACA of $I_{\mathrm{s}}$ fluctuation (a), as well as CCAs of $V_{\mathrm{r}}$ fluctuation (b) and turbulent particle flux $\Gamma_{\mathrm{r}}(\mathrm{c})$, measured at $\Delta r=-2 \mathrm{~cm}$ under $0 \mathrm{~V}$ bias. 

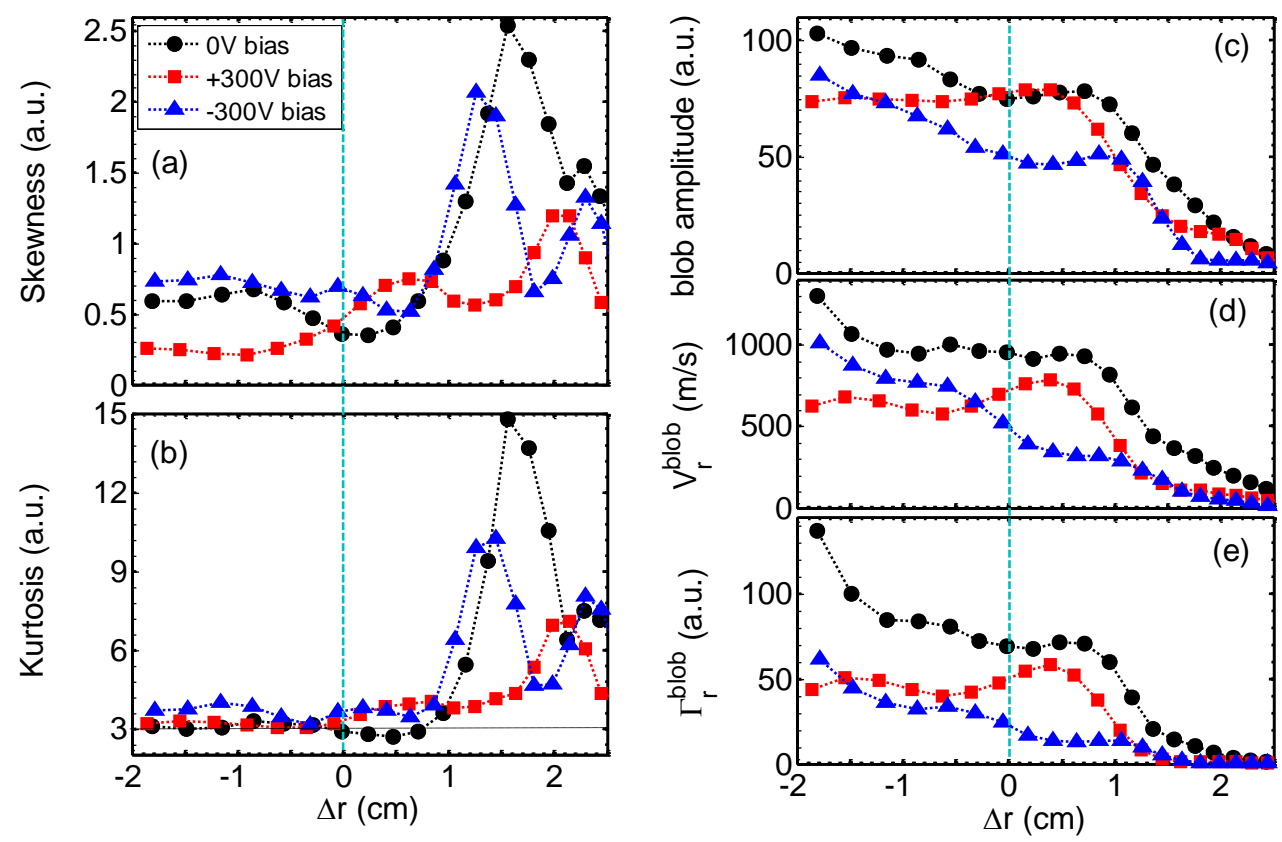

Figure 17. Profiles of skewness (a) and kurtosis (b), as well as the amplitude (c), radial velocity (d) and particle transport (e) related to blob under different bias cases. The vertical lines indicate the position of limiter.
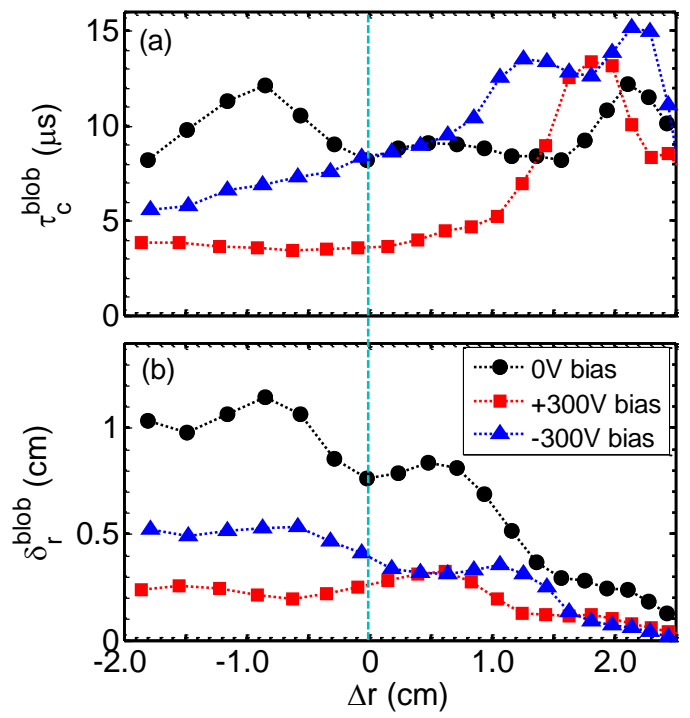

Figure 18. Profiles of blob life time $\tau_{\mathrm{c}}^{\text {blob }}$ (a) and radial size $\delta_{\mathrm{r}}^{\text {blob }}$ (b) under different bias cases. The vertical line indicates the position of limiter.

Moreover, the blob life time $\tau_{\mathrm{c}}^{\text {blob }}$ and radial size $\delta_{\mathrm{r}}^{\text {blob }}$ are all dramatically decreased inside limiter under bias comparing to $0 \mathrm{~V}$ bias case, as shown in figure 18 . The blob life time $\tau_{\mathrm{c}}^{\text {blob }}$ is about $8 \sim 12 \mu$ s under $0 \mathrm{~V}$ bias inside limiter, while decreases to $5 \sim 8 \mu$ s and $3 \sim 4 \mu \mathrm{s}$ under negative and positive bias cases, respectively. Along with the reduction of radial velocity $V_{\mathrm{r}}^{\text {blob }}$ under bias, the resultant radial size of blob $\delta_{\mathrm{r}}^{\text {blob }}$ decreases by a factor of $\sim 2$ under negative bias and of $\sim 4$ under positive bias inside limiter, comparing to the $0 \mathrm{~V}$ bias case. 


\section{Discussions}

According to the results presented above, the responses of plasma to positive and negative bias show both common and different characteristics. The common characteristics mainly include: (1) improvement of global particle confinement of plasma, with increase of central line-averaged density and soft X-ray emission as well as decrease of edge $\mathrm{H}_{\alpha}$ radiation; (2) enhancement of plasma-edge shearing rate of radial electric field $E_{\mathrm{r}}$ and suppression of plasma-edge turbulent particle transport; (3) speed-up of poloidal phase velocity of turbulence $V_{\mathrm{ph}}$ at plasma-edge; (4) increase of poloidal correlation length of turbulence $L_{\mathrm{c} \theta}$ and (5) decrease of radial size of blobs $\delta_{\mathrm{r}}^{\text {blob }}$ at plasma-edge. As revealed by the statistical results in figure 19 (a), the poloidal correlation length $L_{\mathrm{c} \theta}$ increases with the amplitude of poloidal phase velocity $V_{\mathrm{ph}}$. An analysis in different time scales (see figure 19 (b) and (c)) show that, $L_{\mathrm{c} \theta}$ in large time scales (f $<25 \mathrm{kHz}$ ), which covers the frequency regions of LFMs, is obviously larger than that in small scales (f $>25 \mathrm{kHz}$ ). This is particularly obvious for $0 \mathrm{~V}$ and positive bias cases, where LFMs are existed. On the other hand, $L_{\mathrm{c} \theta}$ in both large and small time scales all increase with the amplitude of poloidal phase velocity $V_{\mathrm{ph}}$. These results indicate the robust relationship between the increment of poloidal phase velocity $V_{\mathrm{ph}}$ and the high-coherence (or large- $L_{\mathrm{c} \theta}$ ) behavior of potential fluctuations in the poloidal direction observed under bias. Another statistical result in figure 20 shows that, the radial size of blobs $\delta_{\mathrm{r}}^{\text {blob }}$ has a negative correlation with the amplitude of poloidal phase velocity. This may be due to the increased shearing effect on the filaments from the shear flows, which can be enhanced with greater amplitude of poloidal velocity.
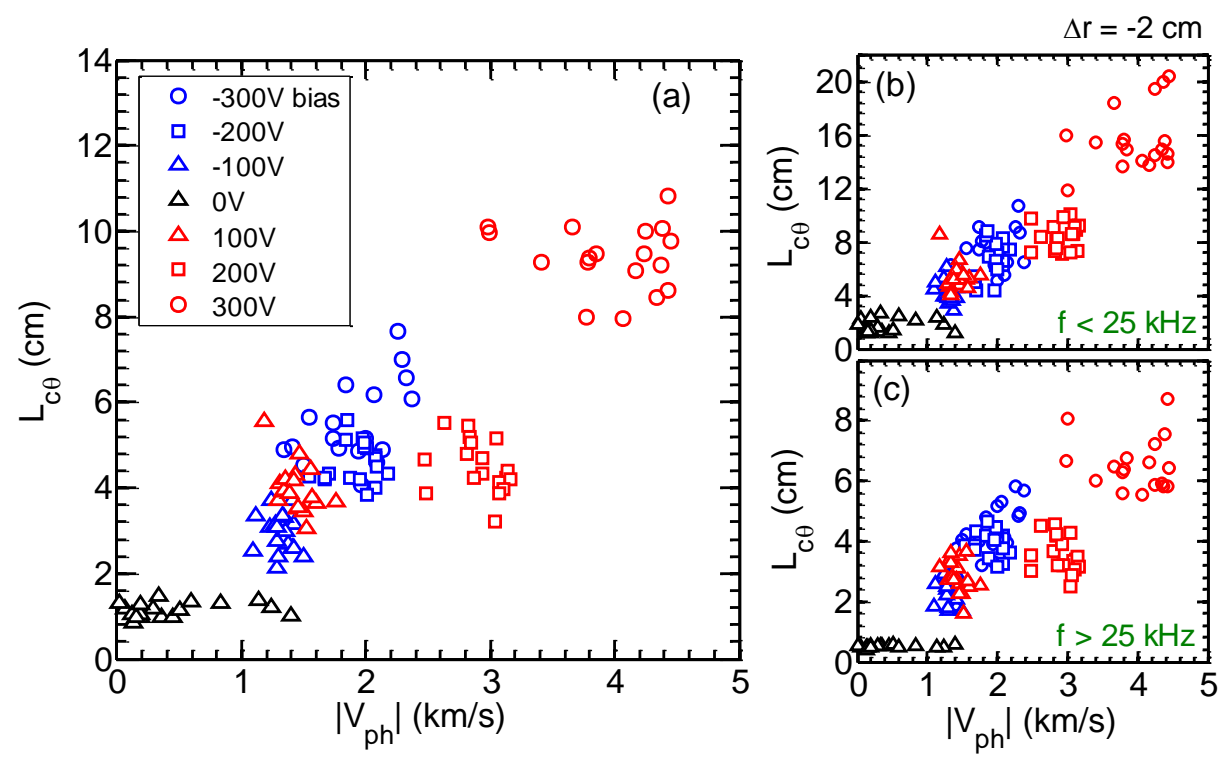

Figure 19. Distributions of poloidal correlation length $L_{\mathrm{c} \theta}$ (a) as well as that in large time scales ( $\mathrm{f}<25 \mathrm{kHz}$ ) (b) and in small time scales (f $>25 \mathrm{kHz}$ ) (c) as functions of absolute value of phase velocity $V_{\mathrm{ph}}$ under different bias conditions at $\Delta \mathrm{r}=-2 \mathrm{~cm}$. 


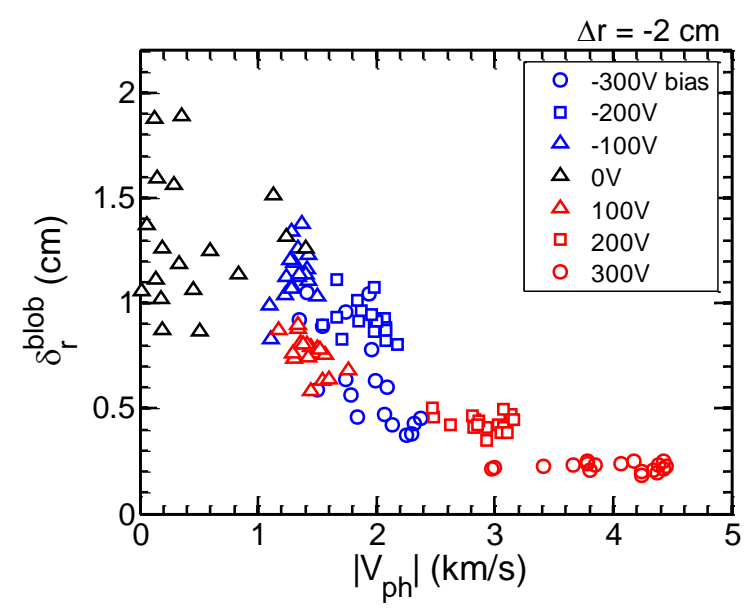

Figure 20. Distribution of radial size of blobs $\delta_{\mathrm{r}}^{\text {blob }}$ as a function of absolute value of phase velocity $V_{\mathrm{ph}}$ under different bias conditions at $\Delta \mathrm{r}=-2 \mathrm{~cm}$.

The main different characteristics of plasma responses concentrate on the potential fluctuation, the behavior of which is sensitive to the polarity of bias. The fluctuation level of floating potential is increased under positive bias at the vicinity of limiter while decreased under negative bias in the whole edge region that measured, comparing to the $0 \mathrm{~V}$ bias case (figure 5 (a)). And spectral analysis in figure 6 shows that, the increase of local potential fluctuation under positive bias is mainly due to the growth of low-frequency spectral power below $50 \mathrm{kHz}$. Together with that, A GAM like low-frequency mode (LFM, $\sim 12 \mathrm{kHz}$ ) characterized as high-coherence and near-zero cross-phase in poloidal direction is existed under positive and $0 \mathrm{~V}$ bias while absent under negative bias at plasma-edge $(\Delta r=-2 \mathrm{~cm})$, and the spectral power of the LFM under positive bias is lower than the case of $0 \mathrm{~V}$ bias (figure 11). These observations may be consistent with the results in ISTTOK [23].

\section{Conclusions}

The experimental results provided above show that both the global particle confinement and the plasma-edge parameters can be modified under bias, with positive or negative polarity. Under negative $(-300 \mathrm{~V})$ bias, the electrode current drawn is lower than the positive $(+300 \mathrm{~V})$ bias case as expected, with a greater variation of plasma state. The absolute value and shearing rate of radial electric field $E_{\mathrm{r}}$ are greatly promoted under bias, with broad shear layers distributed in both polarity cases. Together with that, the local fluctuations and turbulent particle flux are evidently suppressed. The suppression of turbulent particle flux is mainly due to the reduction of fluctuation levels in the positive bias case, and is attributed to the combined role of fluctuation suppression and de-phasing under negative bias, at plasma-edge.

The behavior of potential fluctuation is found to be sensitive to the polarity of bias. That is, the low frequency power density $(<50 \mathrm{kHz})$ of potential fluctuation is increased in the edge 
region under positive bias, with the existence of a peaked low frequency mode $(\sim 12 \mathrm{kHz})$ characterized as high coherence and near-zero poloidal phase shift, which is no found in the negative bias case. The poloidal correlation length of turbulence is greatly enhanced under bias, and shows a positive correlation with the amplitude of poloidal phase velocity, which is mainly driven by the poloidal component of $J_{\mathrm{r}} \times B$ torque in the bias phase. In addition, the influence of bias on the intermittent events (i.e. blobs) and related transport is studied. The intermittency of density fluctuation, which is quantified by the statistical parameters (i.e. skewness and kurtosis), is evidently suppressed under positive bias inside limiter, with an obvious reduction of the characteristic parameters, including the amplitude, radial velocity and the related transport of blob. While under negative bias, the intermittency shows no suppression, and the resulting reduction of characteristic parameters of blob is mainly due to the decrease of fluctuation levels as well as the de-phasing between the fluctuation quantities. Comparing to the $0 \mathrm{~V}$ bias case, the blob radial size is decreased by a factor of $\sim 2$ and $\sim 4$ under negative and positive bias, respectively.

\section{Acknowledgements}

The authors are grateful to Professor C X Yu and Dr L Wang for useful discussions about experimental results and to Mr. B Rao for the helps of the EBS power supply. This work is supported by National Natural Science Foundation of China (No. 10935004 and 10990214) and the Ministry of Science and Technology (Contract No.2011GB109001, 2013GB106001) .

\section{References}

[1] Wagner F et al 1982 Phys. Rev. Lett. 491408

[2] Taylor R J, Brown M L, Fried B D, Grote H, Liberati J R, Morales G J, and Pribyl P 1989 Phys. Rev. Lett. 632365

[3] Weynants R R et al 1992 Nucl. Fusion 32837

[4] Jachmich S, Van Oost G, Weynants R R and Boedo J A 1998 Plasma Phys. Control. Fusion 401105

[5] Boedo J, Gray D, Jachmich S, Conn R, Terry G P, Tynan G, Van Oost G and Weynants R R 2000 Nucl. Fusion 401397

[6] Boedo J A, Gray D S, Terry P W, Jachmich S, Tynan G R, and Conn R W 2002 Nucl.Fusion 42117

[7] Ghosh J, Pal R and Chattopadhyay P K 1999 Rev. Sci. Instrum. 704557

[8] Silva C, Figueiredo H, Nedzelsky I, Goncalves B, Cabral J A C, Varandas C A F and Van Oost G 2004 Plasma Phys. Control. Fusion 46163

[9] Kirnev G S et al 2003 Plasma Phys. Control. Fusion 45337

[10] Nascimento I C et al 2005 Nucl. Fusion 45796 
[11] Stockel J et al 2005 Plasma Phys. Control. Fusion 47635

[12] Roquemore A L, Zweben S J, Kaita R, Marsalsa R J, Bush C E and Maqueda R J 2008 Rev. Sci. Instrum. 79 10F124

[13] Lu H W, Luo J R, Zhong F C, Zha X J and Hu L Q 2012 Eur. Phys. J. D. 66213

[14] Pedrosa M A, Silva C, Hidalgo C, Carreras B A, Orozco R O and Carralero D 2008 Phys. Rev. Lett. 100215003

[15] Manz P, Ramisch M, and Stroth U 2009 Phys. Plasma 16042309

[16] Antoni V, Martines E, Desideri D, Fattorini L, Serianni G, Spolaore M, Tramontin L and Vianello N 2000 Plasma Phys. Control. Fusion 4283

[17] Schaffner D A, Carter T A, Rossi G D, Guice D S, Maggs J E, Vincena S and Friedman B 2012 Phys. Rev. Lett. 109135002

[18] Theiler C, Furno I, Loizu J and Fasoli A 2012 Phys. Rev. Lett. 108065005

[19] Van Oost G et al 2003 Plasma Phys. Control. Fusion 45621

[20] Kasuya N, Itoh K and Takase Y 2003 Nucl. Fusion 43244

[21] Silva C, Nedzelsky I, Figueiredo H, Galvão R M O, Cabral J A C and Varandas C A F 2004 Nucl. Fusion 44779

[22] Xu Y, Jachmich S, Weynants R R, Van Schoor M, Vergote M, Krämer-Flecken A, Schmitz O, Unterberg B and Hidalgo C 2009 Phys. Plasma 16110704

[23] Silva C, Duarte P, Fernandes H and Figueiredo H 2012 Plasma Phys. Control. Fusion 54 085013

[24] Kuznetsov Y K et al 2012 Nucl. Fusion 52063004

[25] Xu Y et al 2011 Nucl. Fusion 51063020

[26] Zhuang G et al 2011 Nucl. Fusion 51094020

[27] Chen Z P, Sun Y, Wang Z J, Yang Z J, Ding Y H and Zhuang G 2012 Plasma Sci. Technol. 141041

[28] Beall J M, Kim Y C and Powers E J 1982 J. Appl. Phys. 533933

[29] Iwama N, Ohba Y and Tsukishima T 1979 J. Appl. Phys. 503197

[30] Zhu M Z, Zhuang G, Wang Z J and Pan Y 2011 Chin. Phys. B 20025204

[31] Powers E J 1974 Nucl. Fusion 14749

[32] Pecseli H L and Trulsen J 1989 Phys. Fluids B 11616

[33] Fujisawa A et al 2007 Nucl. Fusion 47 S718 